\title{
BIODIESEL RENOVÁVEL DERIVADO DE MICROALGAS: AVANÇOS E PERSPECTIVAS TECNOLÓGICAS
}

Claudio M. P. Pereira, Cristiane B. Hobuss, Juliana Villela Maciel, Lizângela R. Ferreira, Francisco B. Del Pino e Márcia F. Mesko*

Centro de Ciências Químicas, Farmacêuticas e de Alimentos, Universidade Federal de Pelotas, Rua Gomes Carneiro, 1, 96010-610 Pelotas - RS, Brasil

Eduardo Jacob-Lopes

Departamento de Tecnologia e Ciência de Alimentos, Universidade Federal de Santa Maria, 97105-900 Santa Maria - RS, Brasil Pio Colepicolo Neto

Departamento de Bioquímica, Instituto de Química, Universidade de São Paulo, CP 26077, 05599-970 São Paulo - SP, Brasil

Recebido em 22/11/11; aceito em 5/7/12; publicado na web em 31/8/12

\begin{abstract}
BIODIESEL DERIVED FROM MICROALGAE: ADVANCES AND PERSPECTIVES. Microalgae are a promising source of raw material for biodiesel production. This review discusses the latest developments related to the application of microalgae biomass for biodiesel production. Characterization of fatty acid of microalgae and comparisons with other sources of raw materials and processes are presented. Furthermore, technological perspectives and approaches for growing microalgae in photobioreactors, microalgal oil extraction techniques, and procedures for synthesizing biodiesel are reviewed.
\end{abstract}

Keywords: microalgae; biofuel; fatty acid.

\section{INTRODUÇÃO}

O biodiesel tem sido objeto de grande atenção nos últimos anos, devido aos impactos e passivos ambientais, além dos altos preços do diesel oriundo de fontes fósseis. O biodiesel é produzido a partir de biomassas renováveis, pela transesterificação de triglicerídeos com alcoóis de cadeia curta, produzindo ésteres monoalquílicos de ácidos graxos de cadeia longa. As principais vantagens deste combustível devem-se ao fato do mesmo ser biodegradável, não tóxico e renovável. Além disso, o biodiesel pode ser misturado ao diesel fóssil em qualquer proporção. ${ }^{1,2}$

A matéria-prima utilizada na síntese de biocombustível contribui para a maior porção nos custos da produção de biodiesel. Em geral, a procura por fontes alternativas e economicamente viáveis tem sido o principal foco de pesquisadores da área. Assim, a fonte ideal para a produção de biocombustível depende principalmente da sua disponibilidade e do seu custo.

O biodiesel pode ser produzido a partir de diferentes fontes, como óleos vegetais (soja, palma, girassol, algodão, amendoim e outros), gordura animal e pelo reuso de óleo (proveniente de fritura). ${ }^{1,3}$ No entanto, a procura por novas fontes para a produção de biocombustíveis não se limita a óleos vegetais e gordura animal, mas também de microalgas. ${ }^{3}$ Estudos recentes indicam que o biodiesel pode ser obtido a partir de microalgas, devido à facilidade de seu cultivo, quantidade intracelular de lipídios, viabilidade de manipulação genética das vias metabólicas, duplicação da biomassa em um curto período de tempo e possibilidade de controlar estas condições. Neste sentido, é emergente a aplicação de microalgas como fonte alternativa para a produção de biodiesel. ${ }^{4,5}$ As microalgas são organismos aquáticos, que crescem em ambientes de águas doce e salgada. Estas podem usar diferentes metabolismos energéticos para manutenção de suas estruturas, como fotossíntese, respiração e fixação/assimilação de nitrogênio, o que torna algumas espécies organismos únicos, com amplo espectro de aplicação tecnológica. ${ }^{6,7}$

A biomassa de microalgas contém três componentes principais:

*e-mail: marcia.mesko@pq.cnpq.br carboidratos, proteínas e lipídios. ${ }^{5,8}$ Sabendo-se que para constituir uma matéria-prima de biodiesel, esta deve ser rica em ácido graxo, uma microalga com um teor de proteínas muito alto e baixo teor de lipídios não seria útil como matéria-prima para biocombustíveis. A maior parte do óleo natural de microalgas está na forma de triacilgliceróis; os ácidos graxos presentes nas algas podem ser de cadeia curta e longa, sendo que os ácidos graxos de cadeia curta são ideais para a produção de biodiesel. ${ }^{5}$ Em particular, alguns ácidos graxos sintetizados por microalgas, tais como o ômega 3 e 6 ( $\omega$-3 e $\omega$ - 6 ), têm um alto valor econômico nas indústrias alimentícia e farmacêutica, pois são os principais precursores de hormônios como prostaglandinas, prostaciclinas, leucotrienos e tromboxanos, em diferentes classes de animais marinhos e terrestres, fundamentais para o desenvolvimento e reguladores fisiológicos. ${ }^{9-11}$

\section{BIOSSÍNTESE DE ÁCIDOS GRAXOS E ACIL GLICERÓIS POR MICROALGAS}

As microalgas, através da fotossíntese, podem sintetizar ácidos graxos a partir de fontes de carbono, como o $\mathrm{CO}_{2}$, e também diretamente a partir de fontes de carbono orgânicos (glicose, acetato, etc.). A composição e a quantidade de lipídios dependem da espécie de microalgas e das condições autotrófica ou heterotrófica de crescimento. ${ }^{10}$

Em especial, a síntese de ácidos graxos pode ser dividida em três etapas principais: síntese de acetil coenzima A (acetil CoA) no citoplasma; síntese de ácido graxo saturado com 16-18 carbonos e posterior dessaturação e elongação da cadeia de carbonos e, síntese dos acil gliceróis (triglicerídeos). ${ }^{10}$

\section{Síntese de acetil CoA}

O intermediário chave para a síntese de acetil CoA nas algas fotossintetizantes é o gliceraldeido fosfato (GAP). A formação de GAP na via fotossintética inclui as reações em fase clara e do ciclo de Calvin, e ocorre nos cloroplastos. O GAP é exportado para o citoplasma e direcionado para a síntese de açúcares ou oxidado através da via glicolítica até piruvato, onde pode produzir energia ou servir 
de substrato para a síntese de compostos como os ácidos graxos. Nas algas heterotróficas, a principal via para obtenção de acetil CoA é a via glicolítica. Os açúcares, incluindo a sacarose, são os principais produtos para estocagem de energia e carbono no citoplasma das células vegetais. ${ }^{10}$

\section{Síntese de ácido graxo saturado com 16-18 carbonos e posterior dessaturação e elongação da cadeia de carbonos}

A síntese de ácidos graxos saturados se dá semelhantemente em plantas superiores e em algas, através de duas enzimas, a acetil CoA carboxilase, que é a enzima regulatória do processo, e o complexo de uma sintase de ácido graxo (AGS).$^{10}$

Em algumas microalgas, as atividades enzimáticas da AGS estão divididas em sete diferentes proteínas (seis enzimas e a proteína carreadora de acilas (ACP)). A acetil CoA é o primeiro composto da síntese e a fonte das unidades de dois carbonos para o alongamento da cadeia. Isto acontece através da incorporação de novas acetilas em um processo cíclico. Nesta primeira reação, catalisado pela AGS, a acetila se condensa com o malonil CoA, formando acetoacetil ligado à proteína carregadora de acilas. Este malonil CoA é originado a partir da reação entre a acetil $\mathrm{CoA}$ e o $\mathrm{CO}_{2}$, catalisada pela acetil CoA carboxilase. Os compostos finais são ácidos graxos saturados com 16-18 carbonos, que se formam através de ciclos da AGS. A síntese de ácidos graxos insaturados nas algas acontece da mesma forma que nas plantas, animais, fungos e bactérias. $\mathrm{O}$ alongamento da cadeia e posterior dessaturação acontece a partir de ácidos graxos com 18 carbonos (saturados ou não) através de alongases e dessaturases, cujo produto final com $\mathrm{C}_{20}-\mathrm{C}_{22}$ carbonos varia de espécie para espécie nas microalgas ${ }^{10}$ (Figura 1).

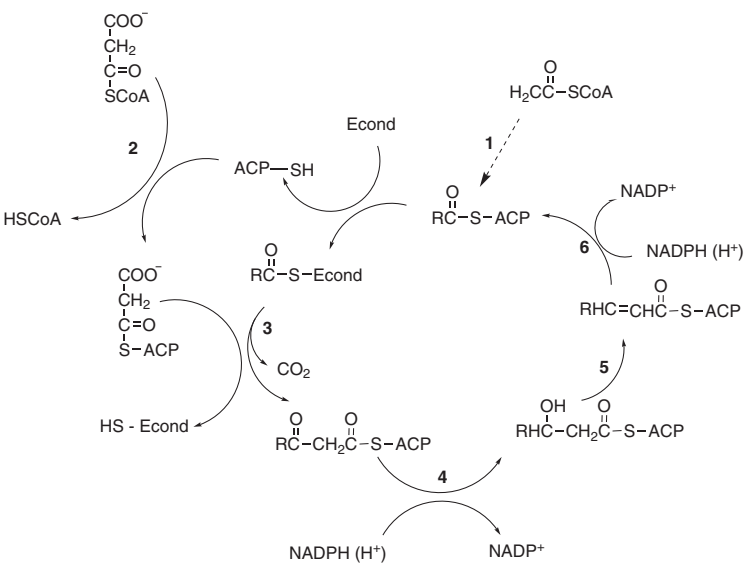

Figura 1. Processo de síntese biológica de ácidos graxos livres. 1- Acetil-CoA-ACP acil transferase. 2- Malonil CoA-ACP acil transferase. 3- $\beta$-cetoacil-ACP. 4- $\beta$-cetoacil-ACP redutase. 5 - $\beta$-hidroxiacil-ACP de-hidrase. 6- Enoil-ACP redutase

\section{Síntese dos acil gliceróis (triglicerídeos)}

As microalgas, assim como outros organismos, são capazes de sintetizar e estocar acil gliceróis como fonte de energia e carbonos. Geralmente, a síntese se dá a partir do L- $\alpha$-fosfoglicerol, oriundo da via glicolítica, e acil CoA. A transferência de resíduos de acilas se dá a partir da acil CoA transferase que forma o ácido lisofosfatídico que, através da L-acilglicerol-3-fosfato-aciltransferase, transfere outra acila formando o ácido L- $\alpha$-fosfatídico (Figura 2). Este composto é a origem dos fosfolipídios ou depois da ação de uma fosfatase e incorporação do terceiro resíduo de acila, através da diacilglicerol aciltransferase, se transforma em triacilglicerol. ${ }^{10}$

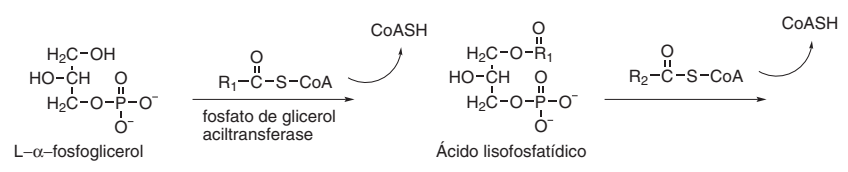

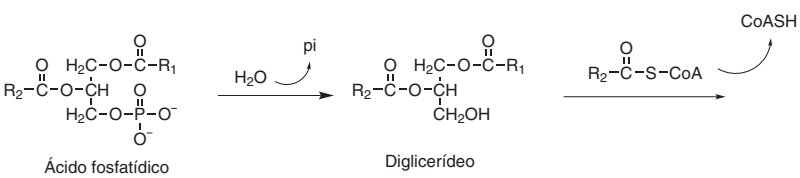$$
\begin{gathered}
\underset{\mathrm{O}}{\mathrm{H}} \mathrm{H}_{2} \mathrm{C}-\mathrm{O}-\stackrel{\mathrm{O}}{\mathrm{C}}-\mathrm{R}_{1} \\
\mathrm{R}_{2}-\mathrm{C}-\mathrm{O}-\mathrm{C} \mathrm{H} \\
\mathrm{H}_{2} \mathrm{C}-\mathrm{O}-\mathrm{O}-\mathrm{C}_{\|}-\mathrm{R}_{3} \\
\text { Triglicerídeo }
\end{gathered}
$$

Figura 2. Biossíntese de triglicerídeos em microalgas

As microalgas crescem muito rapidamente quando comparadas às culturas terrestres. Durante a fase de pico de crescimento, algumas microalgas podem duplicar a cada 3,5 h. Assim, o fato das microalgas crescerem de forma acelerada também as torna uma cultura promissora para uso econômico. ${ }^{12}$

\section{Formas de cultivo de microalgas}

A produção de biomassa microalgal é realizada em biorreatores. Metabolicamente é possível utilizar a rota fotossintética em fotobiorreatores ou a rota respiratória em biorreatores heterotróficos. ${ }^{13,14}$

Em particular, nos sistemas fotossintéticos, a produção de biomassa é realizada através da conversão de dióxido de carbono $\left(\mathrm{CO}_{2}\right)$, energia luminosa e nutrientes inorgânicos. Em termos de configuração, o cultivo fotossintético permite o emprego de sistemas abertos, nos quais os cultivos estão expostos em contato direto com a atmosfera, e sistemas fechados, em que o contato com a atmosfera é significantemente reduzido ou inexistente. Os principais arranjos dos reatores abertos são tanques retangulares, circulares e do tipo raceway. ${ }^{6}$ Nos sistemas fechados, normalmente empregam-se reatores do tipo coluna de bolhas, air-lift e arranjos tubulares espirais. ${ }^{15}$

Os sistemas heterotróficos consistem na conversão de compostos orgânicos, na ausência de energia luminosa, para a produção de biomassa, consequentemente, reatores fechados do tipo coluna de bolhas, air-lift e agitados mecanicamente são empregados nestes cultivos.?

Comparativamente, os aspectos construtivos são mais simples nos sistemas heterotróficos, que operam em ausência de energia luminosa, podendo-se utilizar materiais industriais convencionais na construção dos biorreatores, reduzindo assim significantemente os custos de implementação. ${ }^{16}$ Por outro lado, os sistemas fotossintéticos, baseados na conversão de $\mathrm{CO}_{2}$, permitem a obtenção de créditos de carbono. ${ }^{15}$ Devese considerar que o elevado custo dos compostos orgânicos assimilados por microalgas somente viabiliza os sistemas heterotróficos através da utilização de resíduos industriais, particularmente águas residuárias. ${ }^{17}$ Em termos de produtividade, não existe um padrão global em relação à produção de biomassa nos sistemas heterotróficos e fotossintéticos. Normalmente observa-se que as células crescem mais rapidamente nos cultivos heterotróficos, embora os cultivos fotossintéticos apresentem maiores densidades celulares ao final dos cultivos. Neste sentido, a escolha deverá ser realizada com base na linhagem celular, no equilíbrio entre velocidade de crescimento e concentração celular, que resultará em elevada produtividade celular. ${ }^{18}$

\section{Potencial químico das microalgas}

Há décadas, as microalgas são cultivadas como base comercial para a produção de compostos com alto valor nutricional, além de 
serem utilizadas nas indústrias cosmética e farmacêutica. A necessidade por uma alternativa renovável para a produção de energia que não compita com a produção de alimento foi um dos fatores principais que atraiu a atenção da pesquisa para as microalgas. A vantagem da microalga como fonte renovável de biodiesel tem sido discutida extensivamente por apresentar altos rendimentos de produção, quando comparados às culturas terrestres. Além disso, a pouca ou nenhuma dependência de variações sazonais e, principalmente, sua composição celular com alto rendimento de óleo e a falta de compostos não fermentadores para a produção de biogás fazem das microalgas uma excelente fonte alternativa para a produção de biodiesel. ${ }^{19}$

O potencial fotossintético das microalgas junto com a sua promissora tecnologia de fixação de $\mathrm{CO}_{2}$ é assunto de grande interesse na pesquisa. As microalgas eucariontes e procariontes (cianobactérias) sintetizam lipídios sob condições ambientais adequadas. Estes micro-organismos podem promover vários tipos de combustíveis renováveis, biodiesel derivado de óleo extraído, etanol e metanol, gás natural como metano produzido pela digestão anaeróbica da biomassa algal e bio-hidrogênio produzido fotobiologicamente. ${ }^{12,20,21}$

O cultivo de microalgas para a produção de bioenergia sugere uma cultura com grande quantidade de energia solar, fornecendo luz e $\mathrm{CO}_{2}$ para a célula e condições adequadas dentro do meio. A eficiência desse processo, conhecido como fotoconversão, é limitada pela eficiência da fotossíntese e do anabolismo celular. Em especial, a taxa de respiração e o metabolismo algal diferem de espécie para espécie, dependendo das condições de cultivo e podem, entretanto, ser somente estimadas. ${ }^{19}$

Em estudo realizado por Chisti e colaboradores, ${ }^{5}$ constatou-se que a microalga, quando comparada com outras culturas, se apresenta como excelente alternativa para a produção de biodiesel, devido ao elevado teor de óleo (Tabela 1). ${ }^{5,22}$

A quantidade de óleo produzido por microalgas varia conforme a sua composição bioquímica e do tipo de cultivo utilizado. $\mathrm{O}$ conteúdo lipídico de algumas microalgas está listado na Tabela 2. Como pode ser observado, algumas espécies podem exceder a $70 \%$ de ácidos graxos. ${ }^{2,21}$

Neste sentido, Miao e colaboradores ${ }^{23}$ estudaram o potencial lipídico da Chlorella protothecoides e avaliaram o cultivo da microalga em condições autotróficas e heterotróficas. Observaram que as células heterotróficas alcançaram conteúdo lipídico em torno de 55\%, cerca de quatro vezes maior que em células autotróficas, que apresentaram em torno de $15 \%$ de conteúdo lipídico.

Em outro estudo, Sobczuk e Cristi ${ }^{24}$ reportaram o potencial lipídico da microalga Choricystis minor, que se mostrou uma fonte
Tabela 1. Comparação de diferentes matérias-primas para a produção de biodiesel. Adaptada das refs. 5 e 22

\begin{tabular}{lc}
\hline Fonte de biodiesel & Produtividade óleo (L/ha) \\
\hline Mamona* & $806^{*}$ \\
Pinhão manso & 1892 \\
Milho & 172 \\
Soja & 446 \\
Canola & 1190 \\
Coco & 2689 \\
Óleo de palma & 5950 \\
Microalga a $^{*}$ & 58700 \\
\hline
\end{tabular}

${ }^{a}$ variedades com $30 \%$ óleo (em peso) na biomassa.

promissora para produção de biodiesel. Nos cultivos foi empregado um fotobiorreator de $4 \mathrm{~L}$ e a condição otimizada para a obtenção de uma quantidade maior de lipídios foi em pH 6 e temperatura de cultivo de $30^{\circ} \mathrm{C}$. Além disso, os autores relataram que o isopropanol foi o melhor solvente para extração do óleo, chegando a 29,7 g para cada $100 \mathrm{~g}$ de biomassa seca. Desta forma, os estudos com variáveis luminosas validam as microalgas como fontes sustentáveis para a produção de biocombustíveis. ${ }^{11,12,25-27}$

\section{MÉTODOS DE EXTRAÇÃO DE ÓLEO DE MICROALGA}

Embora a extração lipídica nas microalgas seja relativamente fácil de ser executada, são usados vários processos para aumentar a eficiência e o rendimento, com baixos custos. O óleo pode ser extraído por processos químicos onde solventes como benzeno, éter ou hexano são usados para promover a extração de lipídios. O uso de enzimas é outro método de extração eficaz, que torna o fracionamento do óleo mais fácil. Além destes métodos, o tradicional choque osmótico também é usado para rompimento da alga. Embora seja um método barato e economicamente viável, nem todas as microalgas são rompidas eficientemente por choque osmótico. Um método bastante inovador é a extração com fluído supercrítico, onde o gás $\mathrm{CO}_{2}$ sofre pressão e aquecimento, adquirindo ambas propriedades de líquido e gás. Este fluído liquefeito atua como solvente, extraindo o óleo da matéria-prima. Neste processo é possível capturar mais energia a partir da célula lipídica, favorecendo a conversão, sendo substancialmente melhor quando comparado ao processo convencional. ${ }^{25}$ Segundo Sialve e colaboradores, ${ }^{26}$ os fatores que levam a essa conclusão estão

Tabela 2. Conteúdo de óleo presente em diferentes espécies de microalgas. Adaptada das refs. 2 e 21

\begin{tabular}{|c|c|c|c|}
\hline Microalga & Conteúdo de óleo (\% peso seco) & Microalga & Conteúdo de óleo ( $\%$ peso seco) \\
\hline Botryococcus braunii & $25,0-75,0$ & Neochloris oleoabundans & $35,0-54,0$ \\
\hline Chlorella sp. & $28,0-32,0$ & Nitzschia sp. & $16,0-47,0$ \\
\hline Chlorella vulgaris & $5,0-58,0$ & Phaeodactylum tricornutum & $20,0-30,0$ \\
\hline Crypthecodinium cohnii & 20,0 & Porphyridium cruentum & $9,0-18,8$ \\
\hline Cylindrotheca sp. & $16,0-37,0$ & Scenedesmus obliquus & $11,0-55,0$ \\
\hline Dunaliella primolecta & 23,0 & Scenedesmus sp. & $19,6-21,1$ \\
\hline Dunaliella tertiolecta & $16,7-71,0$ & Schizochytrium sp. & $50,0-77,0$ \\
\hline Euglena gracilis & $14,0-20,0$ & Spirulina maxima & $4,0-9,0$ \\
\hline Isochrysis sp. & $25,0-33,0$ & Spirulina platensis & $4,0-16,6$ \\
\hline Monallanthus salina & $>20,0$ & Tetraselmis suecica & $15,0-23,0$ \\
\hline Nannochloris sp. & $20,0-35,0$ & Tetraselmis sp. & $12,6-14,7$ \\
\hline Nannochloropsis sp. & $31,0-68,0$ & & \\
\hline
\end{tabular}


relacionados à baixa biodegradabilidade da microalga, a sua composição bioquímica e a natureza de sua parede celular.

A conversão de biomassa residual da microalga em biocombustível é tão importante quanto a extração lipídica para gerar biodiesel e, neste contexto, dois tipos de processos de conversão têm sido explorados: termoquímicos e bioquímicos. É importante ressaltar que a retirada de lipídios, acrescida da conversão de biomassa em outros combustíveis, torna o processo com um rendimento próximo a $100 \%$ e as microalgas como um dos organismos mais eficientes na geração de combustíveis não fósseis. ${ }^{9,25,28,29}$

Os processos termoquímicos se dividem em gaseificação, onde a biomassa residual oriunda da extração de lipídios contendo esqueletos carbônicos é convertida a gás por oxidação parcial com ar e oxigênio em temperaturas elevadas; liquefação, que consiste na conversão da biomassa em combustível líquido através de uma reação hidrotérmica; pirólise, onde a produção do biocombustível baseia-se na ruptura da estrutura original da biomassa pela ação do calor em atmosfera com pouco ou nenhum oxigênio; e hidrogenação, que é um processo de redução química a elevadas temperaturas e pressões na presença de catalisador e solvente. ${ }^{9,11,25-29}$

\section{Processos de transesterificação}

No estudo realizado por Huang e colaboradores, ${ }^{10}$ foram apresentadas algumas vantagens e desvantagens dos diferentes métodos de catálise existentes para processos de transesterificação (Tabela 3).

Ehimen e colaboradores ${ }^{30}$ descreveram o efeito de importantes variáveis na reação para produção de biodiesel. A transesterificação in situ sob catálise ácida é efetuada a partir de ácidos graxos provenientes de biomassa algal. Os resultados descritos por esses autores indicam que um aumento na quantidade de álcool e um aumento da temperatura de reação levam a uma otimização na conversão de ésteres metílicos oriundos de ácidos graxos (FAMES). No entanto, a transesterificação in situ efetuada à temperatura ambiente $\left(23^{\circ} \mathrm{C}\right)$, mostrou-se uma exceção ao caso, visto que o equilíbrio na conversão dos FAMES parece se aproximar dos limites assintóticos para tempos de reações superiores a $8 \mathrm{~h}$ para todas as temperaturas investigadas. Contudo, há um fator limitante na conversão, e este se mostrou na quantidade de umidade presente na biomassa algal, visto que, quanto maior o teor de umidade da matéria-prima, mais difícil será a extração de lipídios. Assim, menor será o rendimento de ácido graxo produzido, sugerindo que a biomassa deve ser seca para se ter uma conversão satisfatória. ${ }^{30}$

Em trabalho realizado por Umdu e colaboradores, ${ }^{31}$ há relato de produção de biodiesel a partir da microalga Nannochloropsis oculata. $\mathrm{O}$ processo de transesterificação se dá via suporte sólido $\left(\mathrm{Al}_{2} \mathrm{O}_{3}, \mathrm{CaO}\right.$ e $\mathrm{MgO}$ ) como catalisador. Segundo os autores, a mistura de óxidos mostrou alta atividade na conversão de ésteres, obtendo-se um rendimento de biodiesel em torno de $97 \%$. Aliado a isso, o catalisador utilizado na reação de transesterificação pôde ser empregado por mais duas vezes, tornando o processo economicamente rentável.

Recentemente, Hellingwerf e colaboradores ${ }^{32}$ descreveram a produção de biodiesel através de uma reação fototrófica. Nesta reação, organismos quimiotróficos combinados com moléculas de açúcar $\mathrm{C}_{3}$ gliceraldeido-3-fosfato, como um centro intermediário ligante, levam à formação de uma série de alcoóis de cadeia curta. Segundo os autores, a mesma estratégia pode ser explorada para produção de biodiesel, conforme a composição bioquímica da microalga a ser utilizada no processo.

Em especial, a microalga heterotrófica Schizochytrium limacinum é um micro-organismo capaz de produzir grande quantidade de biomassa com significativo conteúdo lipídico. Johnson e Wen utilizaram o método em dois estágios (extração seguida de transesterificação) e elaboraram uma técnica que permitiu a obtenção de biodiesel de forma direta da biomassa seca ou úmida da microalga Schizochytrium limacinum. $\mathrm{O}$ método em dois estágios resultou em $57 \%$ de biodiesel bruto, com um conteúdo de metil éster de ácidos graxos de $67 \%$. O método direto com biomassa seca levou a um alto rendimento de biodiesel bruto e quando a biomassa úmida foi utilizada, resultou em um rendimento de biodiesel baixo. ${ }^{4}$

Francisco e colaboradore ${ }^{18}$ realizaram a síntese de biodiesel a partir de seis espécies de microalgas: Aphanothece, Chlorella, Dunaliella, Phaeodactilum, Phormidium e Scenedesmus. Parâmetros do biodiesel também foram determinados pelos autores (Tabela 4).

Além disso, deve ser dado destaque para a utilização de técnicas assistidas por ultrassom, que através do efeito da cavitação podem promover reações eficientes, como no caso da obtenção de biodiesel derivado da Chlorella vulgaris. Empregando a metodologia Bligh and Dyer foram encontrados 26,0 g de lipídio para cada $100 \mathrm{~g}$ de biomassa seca. No experimento foi empregada uma sonda de ultrassom de 500 $\mathrm{W}$, proporcionando a obtenção direta do biodiesel. O percentual de ésteres obtido foi de $60 \%$. $^{33}$

Além dos estudos para melhorar os rendimentos das reações de transesterificação, recentemente muitos avanços foram efetuados na área de cultivo e bioprospecção de microalgas, visando a obtenção de biodiesel. Neste sentido, Doan e colaboradores coletaram 21 espécies de microalgas das águas costeiras da Singapura e avaliaram vários parâmetros, como, taxa de crescimento celular, concentração de biomassa, conteúdo lipídico e perfil de ácidos graxos. Em particular, a espécie Nannochloropsis apresentou teores lipídicos que variaram na faixa de 40 a $45 \%$ por peso seco da biomassa. Ainda, os autores relataram que a conversão desses lipídios em biodiesel foi de 25 a $51 \%$. Desta forma, este estudo comprovou que a Nannochloropsis é uma espécie promissora como matéria-prima para biodiesel. ${ }^{34}$

Tabela 3. Comparação entre tecnologias de transesterificação para a produção de biodiesel. Adaptada da ref. 10

\begin{tabular}{|c|c|c|}
\hline Tipo de transesterificação & Vantagens & Desvantagens \\
\hline Catálise química & $\begin{array}{l}\text { Condições reacionais podem ser controladas; } \\
\text { Produção em grande escala; } \\
\text { Processo de produção de baixo custo; } \\
\text { Metanol produzido no processo pode ser reciclado; } \\
\text { Alta taxa de conversão em produto final. }\end{array}$ & $\begin{array}{l}\text { Temperatura reacional relativamente alta; } \\
\text { Processo relativamente complicado; } \\
\text { Processo de eliminação mais complexo; } \\
\text { O processo necessita de muita energia; } \\
\text { A água residual após a lavagem do produto polui o ambiente. }\end{array}$ \\
\hline Catálise enzimática & $\begin{array}{l}\text { Condições reacionais moderadas; } \\
\text { Necessário apenas pequena quantidade de metanol; } \\
\text { Praticamente não produz poluente para o ambiente. }\end{array}$ & $\begin{array}{l}\text { Limitação da enzima na conversão de ácidos graxos de cadeia } \\
\text { pequena; } \\
\text { Produtos químicos no processo de produção são danosos para } \\
\text { a enzima. }\end{array}$ \\
\hline Fluido supercrítico & $\begin{array}{l}\text { Fácil de ser controlado; } \\
\text { É seguro e rápido; } \\
\text { Não polui o ambiente. }\end{array}$ & $\begin{array}{l}\text { Altas temperatura e pressão nas condições reacionais, levando } \\
\text { a altos custos de produção e gastos energéticos. }\end{array}$ \\
\hline
\end{tabular}


Tabela 4. Parâmetros de diferentes espécies de microalgas. Adaptada da ref. 18

\begin{tabular}{|c|c|c|c|c|c|c|}
\hline Fonte & TE $(\%)$ & $\mathrm{NC}$ & IS & II $\left(\mathrm{gI}_{2} 100 \mathrm{~g}^{-1}\right)$ & GI $(\%)$ & $\operatorname{PEFF}\left({ }^{\circ} \mathrm{C}\right)$ \\
\hline Aphanothece & 99,9 & 55,8 & 225,1 & 65,4 & 70,6 & $-4,6$ \\
\hline Chlorella & 99,8 & 56,7 & 217,8 & 65,0 & 74,1 & 4,5 \\
\hline Dunaliella & 99,8 & 52,2 & 220,8 & 83,8 & 98,0 & $-8,4$ \\
\hline Phaeodactilum & 99,9 & 53,7 & 266,1 & 58,7 & 52,7 & $-12,3$ \\
\hline Phormidium & 99,8 & 54,6 & 217,9 & 74,5 & 76,3 & 4,4 \\
\hline Scenedesmus & 99,8 & 56,1 & 217,5 & 68,2 & 67,8 & 20,8 \\
\hline
\end{tabular}

TE - Teor de Ésteres; NC - Número de Cetano; IS - Índice de Saponificação; II - Índice de Iodo; GI - Grau de Insaturações; PEFF - Ponto de Entupimento de Filtro a Frio.

Yang e colaboradores estudaram diferentes meios de cultivo para microalgas, a fim de bioprospectar um meio que proporcionasse um aumento de biomassa algal. Avaliaram o ciclo da água, sua reciclagem e suas diferentes características, como, água do mar e água de efluente, além do uso de fotobiorreatores. Os estudos foram efetuados em um tanque, a fim de se avaliar a produção de microalgas em larga escala. Segundo os autores, a água do mar, a água derivada de efluente e a água reciclada de cultivo apresentaram bons resultados, pois nesses meios estavam disponíveis quantidades significativas de nutrientes. $\mathrm{O}$ estudo concluiu que uma forma alternativa e eficaz para produção de biomassa algal são os fotobiorreatores que, além de possibilitarem a reciclagem da água, permitem um controle efetivo das condições do cultivo. Todavia, a utilização de fotobiorreatores apresenta um custo elevado, o que está diretamente relacionado ao custo do produto final. Desta forma, o cultivo de microalgas em lagoas fechadas ainda é uma alternativa promissora. ${ }^{35}$ Resultados expressivos de cultivos em lagoas fechadas foram alcançados por Moazami e colaboradores, empregando cultivos de Nannochloropsis $\mathrm{sp} .46 \mathrm{~g} \mathrm{~L}^{-1} \mathrm{~m}^{-2}$ e percentual lipídico de 52\%, com 14 dias de cultivo. ${ }^{36}$

Uma avaliação em larga escala também foi recentemente efetuada, incluindo a determinação das necessidades biológicas para macronutrientes (fosfato, nitrato e sulfato) e a determinação da produtividade máxima de biomassa, através do controle da incisão de luz com fotobiorreatores. A maior concentração de lipídios totais foi alcançada com Neochloris oleoabundans, (25-37\% de peso seco), enquanto que o maior teor de triacilglicerídeos (TAG) foi encontrado na Chlorella vulgaris (11-14\% de peso seco). Estas duas espécies apresentaram produtividades semelhantes em TAG. ${ }^{37}$

Almejando a obtenção de biodiesel derivado dos óleos extraídos da Scenedesmus sp. e Nannochloropsis, onde as taxas de ácidos graxos podem chegar até $65 \%$, utilizou-se uma razão molar de 12:1 de metanol/óleo e um percentual catalítico de $2 \%$ de $\mathrm{KOH}$ para a transesterificação, sendo obtida uma conversão de $100 \% .^{38}$

Variações de processos de extração de lipídios derivados de microalgas produtoras de óleo, como a Chlorella vulgaris e Chlorella pyrenoidosa, ainda estão sendo pesquisadas, a fim de se explorar a biodisponibilidade celular de lipídios. ${ }^{39,40}$ A moagem e trituração têm sido uma alternativa, acompanhadas de sonicação, ação enzimática ou, ainda, micro-ondas, que são ferramentas potenciais, que podem otimizar metodologias para o aproveitamento da matéria-prima para posterior obtenção de biodiesel. ${ }^{40}$

\section{PARÂMETROS FÍSICO-QUÍMICOS DO BIODIESEL}

Tendo em vista que o biodiesel é uma alternativa promissora apropriada para substituir os combustíveis fósseis, Johnson e colaboradores avaliaram parâmetros como glicerol livre, número de acidez, conteúdo saponificável, viscosidade, material particulado, dentre outros, no biodiesel obtido a partir do óleo da microalga
Schizochytrium limanicum. A partir desta avaliação, os autores enfatizaram que a biomassa algal não só é uma fonte sustentável do ponto de vista sócio-econômico para a produção de biodiesel, como também o produto obtido possui as características físico-químicas apropriadas para utilização como fonte de bioenergia. ${ }^{4}$

Na Tabela 5 é apresentada uma comparação entre os parâmetros físico-químicos do biodiesel obtido a partir de microalgas com as propriedades do óleo diesel. As propriedades foram avaliadas de acordo com as normas estabelecidas pela American Society for Testing and Materials (ASTM). Como pode ser observado, o biodiesel obtido de microalgas apresentou características semelhantes às do diesel. ${ }^{4}$

Tabela 5. Comparação entre os parâmetros físico-químicos do biodiesel obtido a partir de microalgas com as propriedades do óleo diesel, de acordo com as especificações da ASTM. Adaptada da ref. 4

\begin{tabular}{lccc}
\hline Propriedades & $\begin{array}{c}\text { Biodiesel de } \\
\text { microalgas }\end{array}$ & $\begin{array}{c}\text { Óleo } \\
\text { diesel }\end{array}$ & $\begin{array}{c}\text { Normas } \\
\text { ASTM }\end{array}$ \\
\hline Densidade $\left(\mathrm{kg} \mathrm{L}^{-1}\right)$ & 0,864 & 0,838 & $0,84-0,90$ \\
Viscosidade $\left(\mathrm{mm}^{2} \mathrm{~s}^{-1}, 40^{\circ} \mathrm{C}\right)$ & 5,2 & $1,9-4,1$ & $3,5-5,0$ \\
Ponto de fulgor $\left({ }^{\circ} \mathrm{C}\right)$ & 115 & 75 & mín 100 \\
Ponto de solidificação $\left({ }^{\circ} \mathrm{C}\right)$ & -12 & $-50-10$ & -- \\
Acidez $\left(\mathrm{mg} \mathrm{KOH} \mathrm{g}^{-1}\right)$ & 0,374 & máx 0,5 & máx 0,5 \\
Poder calorífico $\left(\mathrm{J} \mathrm{kg}^{-1}\right)$ & 41 & $40-45$ & -- \\
\hline
\end{tabular}

\section{Contaminantes inorgânicos em biodiesel}

Para a utilização do biodiesel como fonte de energia alternativa é necessária, também, uma avaliação da concentração dos metais que fazem parte de sua composição ou que foram adicionados na etapa de transesterificação. ${ }^{41-43}$ Assim, o monitoramento de metais, como K e Na, torna-se indispensável, uma vez que são adicionados na forma de hidróxido e agem como catalisadores no processo de síntese do biodiesel. Esses elementos podem estar presentes na forma de sólidos abrasivos ou de sabões insolúveis, que podem contribuir para danos e corrosão nas peças do motor, afetando seu desempenho e, consequentemente, causando danos ambientais.

Outros metais podem estar presentes na composição final do biodiesel, pois a matriz utilizada na síntese pode assimilar os metais provenientes do solo e da água. ${ }^{41,44,45}$ Neste sentido, muitas normas estão sendo elaboradas e/ou adaptadas das existentes, principalmente, para óleo diesel. Elementos como $\mathrm{Na}, \mathrm{K}, \mathrm{Mg}$, Ca e P possuem limites máximos especificados pela Agência Nacional do Petróleo, Gás Natural e Biocombustíveis (ANP n 7 de 19/3/2008), ${ }^{43}$ bem como são definidos os métodos de análises. De acordo com o especificado na resolução $\mathrm{ANP}^{\circ}{ }^{\circ}$, devem-se utilizar técnicas como a espectrometria de absorção atômica (AAS) e a espectrometria de emissão óptica com plasma indutivamente acoplado (ICP OES), seguindo as normas NBR 
15553, NBR 15554, NBR 15555 e NBR 15556. Entretanto, os métodos referenciados necessitam de validação para as matérias-primas não previstas, bem como na rota de produção etílica. ${ }^{43}$

Além disso, é necessária a avaliação das concentrações de outros elementos metálicos que, quando presentes em concentrações indesejáveis, podem alterar a qualidade do biodiesel causando poluição. Dentre esses elementos destacam-se $\mathrm{Cd}$ e $\mathrm{Pb}$ que, mesmo em baixas concentrações podem alterar a estabilidade do biocombustível, além de apresentarem características toxicológicas extremamente danosas ao meio ambiente. . $^{3,41}$

Desta forma, considerando os aspectos de qualidade do biodiesel e o impacto ambiental causado pelo seu uso, visto que é utilizado como forma alternativa de geração de energia, é relevante o controle dos elementos químicos presentes na sua composição.

\section{CONCLUSÃO}

Dentro deste contexto, sabe-se que as microalgas são potencialmente sustentáveis para a produção de biocombustíveis. Estas constituem um grupo peculiar de micro-organismos fotossintéticos que crescem rapidamente devido à sua estrutura simples, o que ressalta a viabilidade do seu uso como fonte para produção de bioenergia. Sua produção pode satisfazer a demanda crescente de energia que, por sua vez, não compromete a oferta de alimentos. Nesse sentido, a evolução das pesquisas, incluindo os avanços com relação aos meios de cultivo mais eficientes, como o uso de fotobiorreatores, tem sido de interesse global.

O processo de separação da biomassa de microalgas, secagem, extração e conversão do óleo em biodiesel e outras tecnologias de processamento são importantes para a viabilidade da implementação comercial do biodiesel de microalgas. Além disso, a análise de contaminantes orgânicos e metálicos, e a avaliação dos parâmetros físico-químicos garantem a qualidade do produto obtido, tornando-o uma alternativa viável para a obtenção de energia.

\section{REFERÊNCIAS}

1. Knothe, G.; van Gerpen, J.; Krahl, J.; Manual de Biodiesel, Edgard Blucher: São Paulo, 2006; Vicente, G.; Martinez, M.; Aracil, J.; Bioresour. Technol. 2004, 92, 297.

2. Khan, S. A.; Rashmi; Hussain, M. Z.; Prasad, S.; Banerjee, U. C.; Renew. Sust. Energ. Rev. 2009, 13, 2361.

3. Ma, F.; Hanna, M. A.; Bioresour. Technol. 1999, 70, 1.

4. Johnson, M. B.; Wen, Z.; Energy Fuels 2009, 23, 5179.

5. Chisti, Y.; Biotechnol. Adv. 2007, 25, 294.

6. Borowitzka, M. A.; J. Biotechnol. 1999, 70, 313.

7. Queiroz, M. I.; Lopes, E. J.; Zepka, L. Q.; Bastos, R. G.; Goldbeck, R.; Bioresour. Technol. 2007, 98, 2163.

8. Gressler, V.; Yokoya, N. S.; Fujii, M. T.; Colepicolo, P.; Mancini, J. F.; Torres, R. P.; Pinto, E.; Food Chem. 2010, 120, 585.

9. Pyle, D. J.; Garcia, R. A.; Wen, Z.; J. Agric. Food Chem. 2008, 56, 3933.

10. Huang, G.; Chen, F.; Wei, D.; Zhang, X.; Chen, G.; Appl. Energy 2010, 87,38 .

11. Guschina, I. A.; Harwood, J. L.; Prog. Lipid Res. 2006, 45, 160.

12. Brennan, L.; Owende, P.; Renew. Sust. Energ. Rev. 2010, 14, 557.

13. Rodolfi, L.; Zittelli, G. C.; Bassi, N.; Padovani, G.; Biondi, N.; Bonini, G.; Tredici, M. R.; Biotechnol. Bioeng. 2009, 102, 100.

14. Li, X.; Xu, H.; Wu, Q.; Biotechnol. Bioeng. 2007, 98, 764.
15. Jacob-Lopes, E.; Revah, S.; Hernández, S.; Shirai, K.; Franco, T. T.; Chem. Eng. J. 2009, 153, 120.

16. Ugwu, C. U.; Aoyagi, H.; Uchiyama, H.; Bioresour. Technol. 2008, 99 , 4021.

17. Zepka, L. Q.; Jacob-Lopes, E.; Goldbeck, R.; Queiroz, M. I.; Chem. Eng. Process. 2008, 47, 1305.

18. Francisco, E. C.; Neves, D. B.; Jacob-Lopes, E.; Franco, T. T.; J. Chem. Technol. Biotechnol. 2010, 85, 395.

19. Lehr, F.; Posten, C.; Curr. Opin. Biotechnol. 2009, 20, 280.

20. Roessler, P. G.; Brown, L. M.; Dunahay, T. G.; Heacox, D. A.; Jarvis, E. E.; Schneider, J. C.; Talbot, S. G.; Zeiler, K. G.; ACS Symp. Ser. 1994, $566,255$.

21. Mata, T. M.; Martins, A. A.; Caetano, N. S.; Renew. Sust. Energ. Rev. 2010, 14, 217.

22. Chechetto, R. G.; Siqueira, R.; Gamero, C. A.; Rev. Ciênc. Agron. 2010 , $41,546$.

23. Miao, X.; Wu, Q.; Bioresour. Technol. 2006, 97, 841.

24. Sobczuk, T. M.; Chisti, Y.; J. Chem. Technol. Biotechnol. 2010, 85, 100.

25. Amin, S.; Energy Convers. Manage. 2009, 50, 1834.

26. Sialve, B.; Bernet, N.; Bernard, O.; Biotechnol. Adv. 2009, 27, 409.

27. Chisti, M. Y.; J. Ramsay Soc. 1980, 27, 24.

28. Nagle, N.; Lemke, P.; Appl. Biochem. Biotechnol. 1990, 24, 355.

29. McKendry, P.; Bioresour. Technol. 2002, 83, 47.

30. Ehimen, E. A.; Sun, Z. F.; Carrington, C. G.; Fuel 2010, 89, 677.

31. Umdu, E. S.; Tuncer, M.; Seker, E.; Bioresour. Technol. 2009, 100, 2828.

32. Hellingwerf, K. J.; Mattos, M. J. T.; J. Biotechnol. 2009, $142,87$.

33. Hobuss, C. B.; Rosales, P. F.; Venzke, D.; Souza, P. O.; Gobbi, P. C.; Gouvea, L. P.; Santos, M. A. Z.; Pinto, E.; Jacob-Lopes, E.; Pereira, C. M. P.; Rev. Bras. Farmacogn. 2011, 21, 361.

34. Doan, T. T. Y.; Sivaloganathan, B.; Obbard, J. P.; Biomass Bioenergy 2011, 35, 2534

35. Yang, J.; Xu, M.; Zhang, X.; Hu, Q.; Sommerfeld, M.; Chen, Y.; Bioresour. Technol. 2011, 102, 159.

36. Moazami, N.; Ashori, A.; Ranjbar, R.; Tangestani, M.; Eghtesadi, R.; Nejad, A. S.; Biomass Bioenergy 2012, 39, 449.

37. Pruvost, J.; Vooren, G. V.; Gouic, B. L.; Couzinet-Mossion, A.; Legrand, J.; Bioresour. Technol. 2011, 102, 150.

38. Chen, L.; Liu, T.; Zhang, W.; Chen, X.; Wang, J.; Bioresour. Technol. 2012, 111, 208

39. D’Oca, M. G. M.; Viêgas, C. V.; Lemões, J. S.; Miyasaki, E. K.; MorónVillarreyes, J. A.; Primel, E. G.; Abreu, P. C.; Biomass Bioenergy 2011, 35,1533 .

40. Zheng, H.; Yin, J.; Gao, Z.; Huang, H.; Ji, X.; Dou, C.; Appl. Biochem. Biotechnol. 2011, 164, 1215.

41. Dorado, M. P.; Ballesteros, E.; Almeida, J. A.; Schellert, C.; Lohrlein, H. P.; Krause, R.; Trans. ASAE 2002, 45, 519.

42. Lobo, F. A.; Goveia, D.; Oliveira, A. P.; Romão L. P. C.; Fraceto, L. F.; Dias Filho, N. L.; Rosa, A. H.; Fuel 2011, 90, 142.

43. Agência Nacional do Petróleo, Gás Natural e Biocombustíveis (ANP), Resolução n. ${ }^{\circ} 07 /$ 2008. http://www.anp.gov.br/, acessada em Junho 2009.

44. Soares, B. M.; Vieira, A. A.; Lemões, J. S.; Santos, C. M. M.; Mesko, M. F.; Primel, E. G.; D’Oca, M. G. M.; Duarte, F. A.; Bioresour. Technol. 2012, 110, 730 .

45. Oliveira, A. P.; Villa, R. D.; Antunes, K. C. P.; Magalhães, A.; Silva, E. C.; Fuel 2009, 88, 764 . 